\title{
A Gyro-Free System for Measuring the Parameters of Moving Objects
}

\author{
Dimitar Dichev ${ }^{1}$, Hristofor Koev ${ }^{1}$, Totka Bakalova ${ }^{2}$, Petr Louda ${ }^{3}$ \\ ${ }^{1}$ Department of Machine and Precision Engineering, Faculty of Machine and Precision Engineering, \\ Technical University of Gabrovo, 4 Hadji Dimitar Street, 5300 Gabrovo, Bulgaria, \\ dichevd@abv.bg, koevh@abv.bg \\ ${ }^{2}$ Institute for Nanomaterials, Advanced Technologies and Innovation, Technical University of Liberec, \\ Studentska st. 2, 46117 Liberec, Czech Republic, tbakalova@seznam.cz \\ ${ }^{3}$ Department of Material Science, Technical University of Liberec, 2 Studentska Street, \\ 46117 Liberec, Czech Republic, petr.louda@tul.cz
}

\begin{abstract}
The present paper considers a new measurement concept of modeling measuring instruments for gyro-free determination of the parameters of moving objects. The proposed approach eliminates the disadvantages of the existing measuring instruments since it is based, on one hand, on a considerably simplified mechanical module, and on the other hand, on the advanced achievements in the area of nanotechnologies, microprocessor and computer equipment. A specific measuring system intended for measuring the trim, heel, roll, and pitch of a ship has been developed in compliance with the basic principles of this concept. The high dynamic accuracy of this measuring system is ensured by an additional measurement channel operating in parallel with the main channel. The operating principle of the additional measurement channel is based on an appropriate correction algorithm using signals from linear MEMS accelerometers. The presented results from the tests carried out by means of stand equipment in the form of a hexapod of six degrees of freedom prove the effectiveness of the proposed measurement concept.
\end{abstract}

Keywords: Gyro-free measuring system, Kalman filter, micro-electro-mechanical system (MEMS), dynamic errors.

\section{INTRODUCTION}

$\mathrm{T}$ HE CURRENT STAGE of development of metrology and measuring equipment is distinguished for the transition from measuring quantities constant within time to measuring variable quantities. This transition is caused by a number of reasons but there are two main reasons [1]. The first one is related to the advanced level of science and technology today and the possibilities for using the hightech achievements in the area of microprocessor and computer equipment for real measuring procedures. The second one refers to the continuous improvement of present means of transport (ships, aircrafts, road transport, etc.) in relation to their speed, safety, comfort, and so on.

In this regard the development and improvement of the measuring equipment for determining the parameters that characterize the time-space position, the motion mode, etc. of the above means of transport are very topical. The control effectiveness of these moving objects depends on the quality (accuracy, reliability, form, and rate of presentation) of the measurement information [2].

To ensure a specific orientation of the above moving objects and to control their motion, measuring instruments providing the required information must be mounted onboard. These instruments must include devices modeling the basic coordinate system [3, 4]. This allows us to determine the position of the moving object when rotating around its center of mass, as well as when moving along with the latter. In addition, it enables us to keep the motion direction set. Therefore, part of the measuring instruments mounted on moving objects must possess properties that ensure continuous storage of particular directions connected with the Earth.
One of those characteristics, which is mandatory for the orientation system of most moving objects, is the local vertical. There are different methods and tools for building and keeping the local vertical in measurement mode $[5,6]$. The functional elements constituting the vertical play a secondary role in organizing the overall metrological structure of the measuring instruments. Hence, they are considerably important when forming the qualitative characteristics of the measuring instruments and systems in this area.

Measuring instruments built on the properties of the gyroscope are widely spread in metrology [7]. This is mainly due to the physical nature of the gyroscope that ensures its stability in relation to the inertial actions caused by the motion of the object. Therefore the dynamic accuracy of the measuring instruments built on this method is guaranteed by stabilizing the vertical in the inertial space. Under the conditions of dynamic actions these instruments provide relatively high accuracy, which reaches dynamic error values up to several tenths of a degree for the best samples [5]. On the other hand, the measuring instruments built on the basis of gyro-verticals are distinguished for a number of disadvantages such as a sophisticated design, less reliability under extreme conditions, requirement of special systems ensuring the gyro-vertical operation; large sizes, high prices, etc. [6], thus limiting, to a great extent, their application.

Current achievements in science and technology provide good perspectives for developing measuring instruments of a new generation, that possess better qualitative features and metrological characteristics. The actual development and improvement of such measuring instruments is first and 
foremost based on the fast advancement of nanotechnologies [8], microprocessor and computer equipment.

Therefore, today new measuring systems whose improved accuracy parameters in dynamic measurement mode are formed on the basis of new measurement concepts and signal processing algorithms can be implemented. On the basis of their improved characteristics, they can more effectively replace the current measuring instruments based on the gyroscopic principle of vertical stabilization.

\section{A CONCEPT OF MODELING MEASURING INSTRUMENTS}

The proposed measurement concept can be successfully used for modeling instruments that measure the angular position of moving objects in relation to the basic coordinate system, as well as their dynamic fluctuations around their instantaneous axis of rotation. It is designed for developing new measuring instruments in this area since it is based on a different approach targeting the elimination of the dynamic error caused by the deviation of the inertial components that model the vertical from the inertial space in real time rather than their stabilization. This modeling concept can overcome the disadvantages of the existing measuring instruments as it is based, on one hand, on a very simplified mechanical module, and on the other hand, on the possibilities of modern measuring equipment in the area of dynamic measurements. In addition, it is based on successfully integrated processing algorithms [8, 9, 10] intended for eliminating the dynamic error [9, 11].

The block diagram illustrating the operating principle of the measuring systems developed according to the concept of the present approach is shown in Fig.1. In general, it consists of a main measurement channel, an additional channel, interfaces to connect with a computer, and program modules for processing and presenting measurement information.

The main channel is used for measuring the current values of the angles determining the position of the moving object in the basic coordinate system. It is based on the gyro-free principle of vertical modeling and a very simplified design, which results in considerably reducing the magnitude of the instrumental errors.

However, this way of modeling the vertical leads to its instability in the inertial space. Under inertial actions caused by the motion and fluctuations of the object, this instability determines the deviation of the elements modeling the vertical from the actual direction of the vertical. All this results in a dynamic error which in some cases can reach inadmissibly high values [12]. Therefore, the proposed concept envisages a procedure eliminating the current values of the dynamic error from the measurement result.

The procedure related to the obtaining of the measurement information required for determining the current values of the dynamic error is implemented in the additional channel (Fig.1.). The latter operates in parallel with the main channel, which provides an opportunity to eliminate the dynamic error from the measurement result in real time. The structure of the additional channel and the type of devices constituting it are specified on the basis of the selected model for determining the current values of the dynamic error and the algorithm for correcting the signal from the main measurement channel.

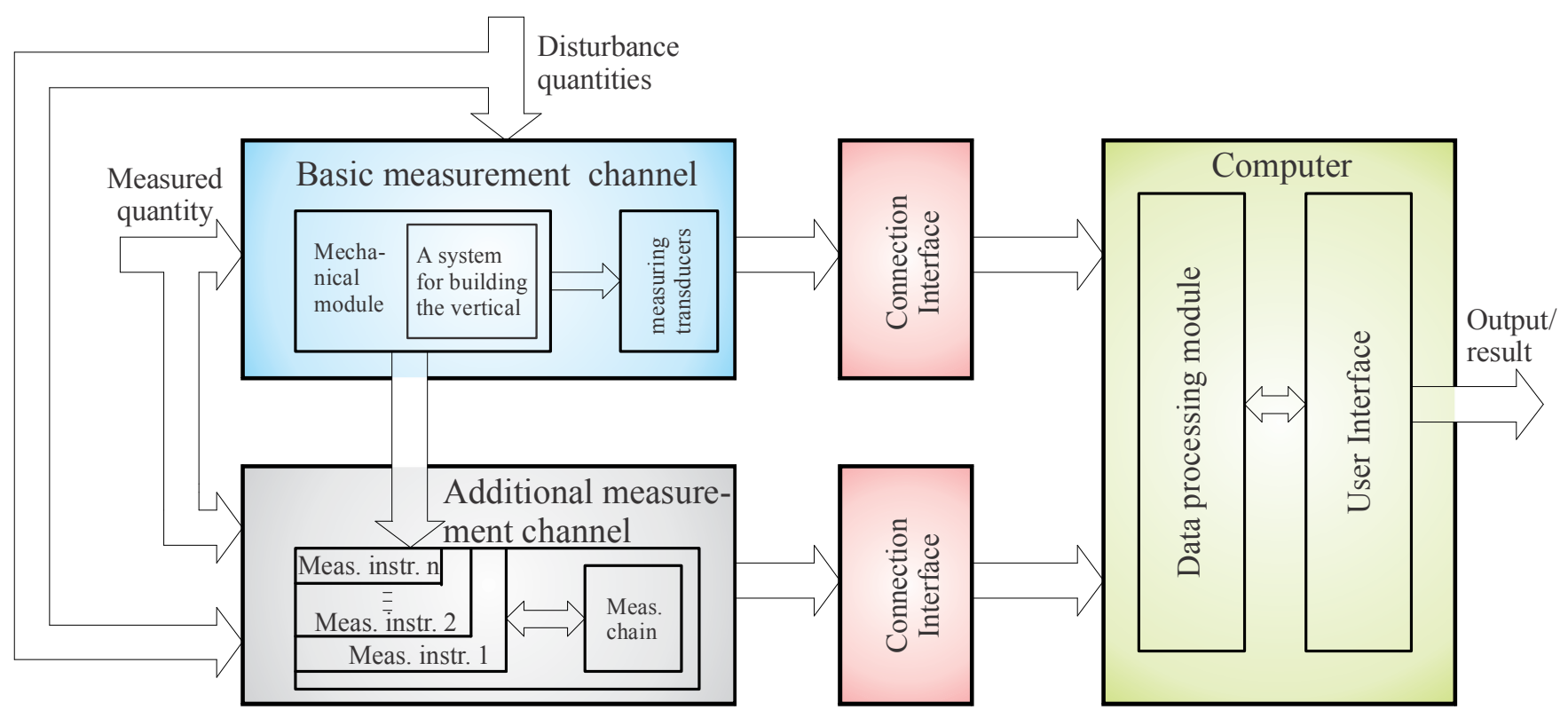

Fig.1. Block diagram illustrating the principle of modeling of measuring systems. 


\section{A MEASURING SYSTEM FOR DETERMINING THE HEEL AND TRIM OF A MOVING OBJECT}

To illustrate the characteristics of the proposed concept, a specific measuring system developed in compliance with the principles of this concept is presented. The system is designed for measuring the roll, pitch, heel, and trim of a ship. Its block diagram is shown in Fig.2.

The basic concept of the present measuring system is focused on the simplified design of the vertical in the form of a physical pendulum (Fig.3.). This comparatively simple design of the mechanical module, consisting of a small number of elements, results in limiting the magnitude of the instrumental error. The body 1 of the mechanical module is fixed to the ship. By means of an appropriate suspension system 2, a system for modeling the vertical 3 is mounted on the body. The latter consists of an outer frame 1 and inner frame 2, connected in series by cylindrical joints (Fig.4.).
A physical pendulum of two degrees of freedom 3 is attached to the inner frame (Fig.4.). The two frames have interperpendicular axes of rotation, which intersect at one point. The measurement information about the heel and trim angles is obtained from identical absolute encoders 4 and 5, mounted on the respective measurement axis.

The absolute encoders 4 and 5 used in the model are intended for transforming the angular displacements in coded electrical signals corresponding to the absolute position of the shaft. The application of differential parallel scanning of each division of the rotating scale in Gray code eliminates the errors due to interferences and provides a wide operating temperature range. The absolute encoders are distinguished for their high accuracy, high noise immunity, fast response, wide range of supply voltage, and small size.

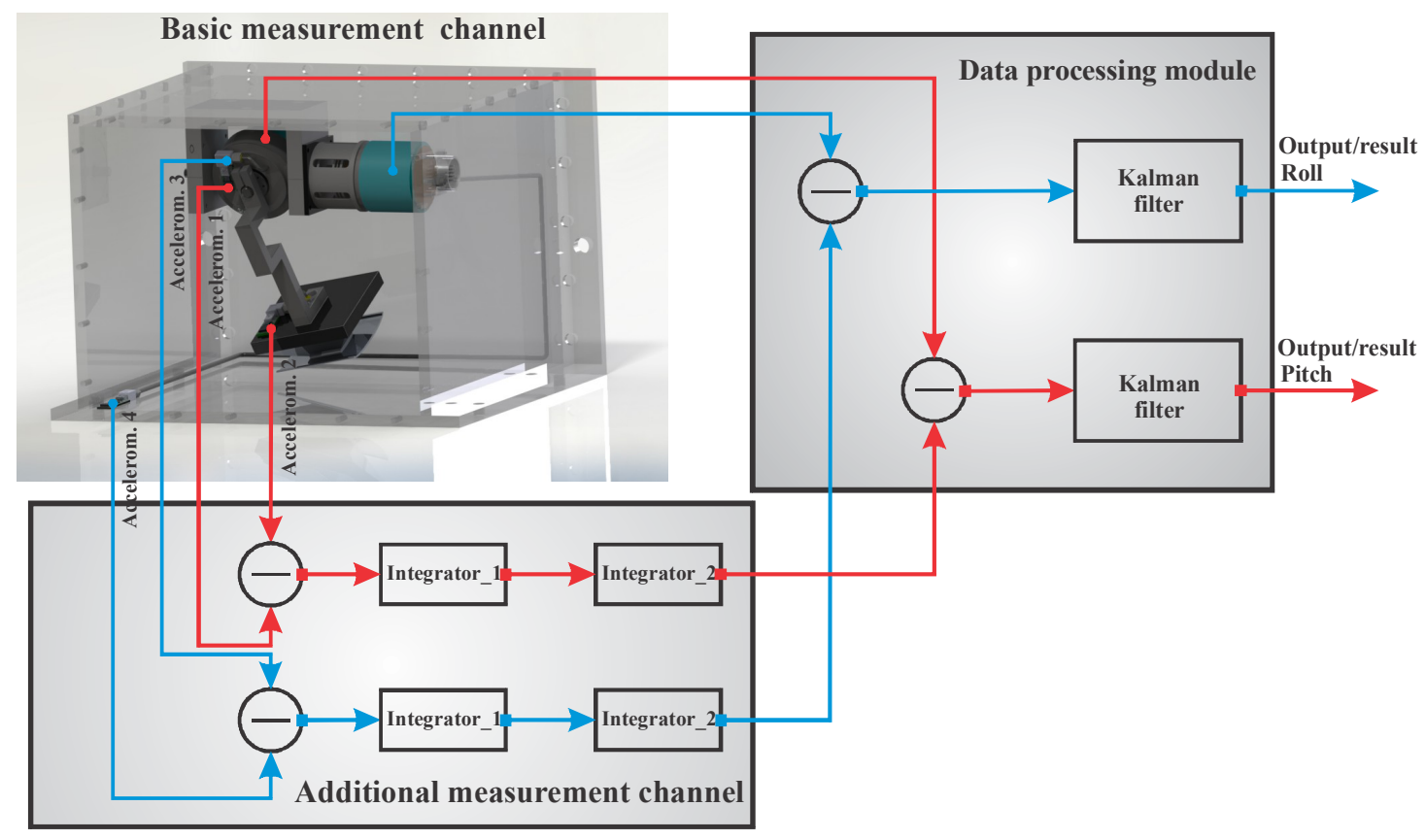

Fig.2. Block diagram of the measuring system.

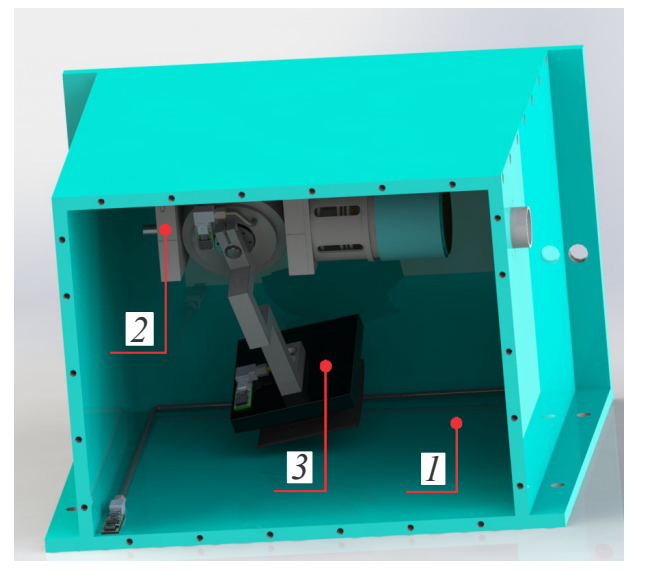

Fig.3. Design model of the main measurement channel.

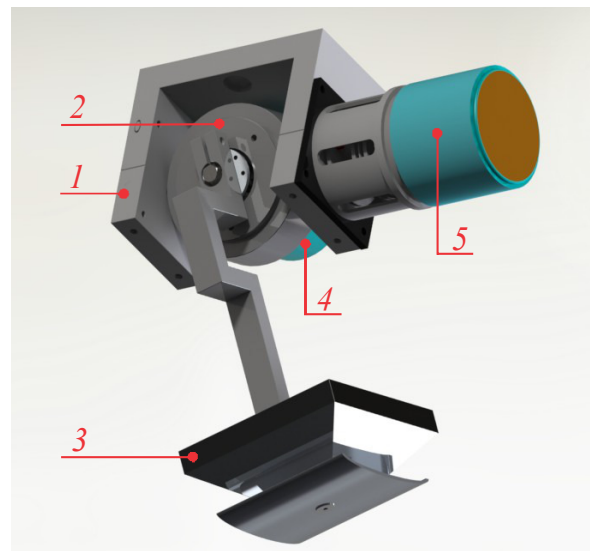

Fig.4. System modeling the vertical of the device. 1 - outer frame; 2 - inner frame; 3 - physical pendulum; 4 and 5 - absolute encoders of FKP 13.A type and 213-bit resolution. 


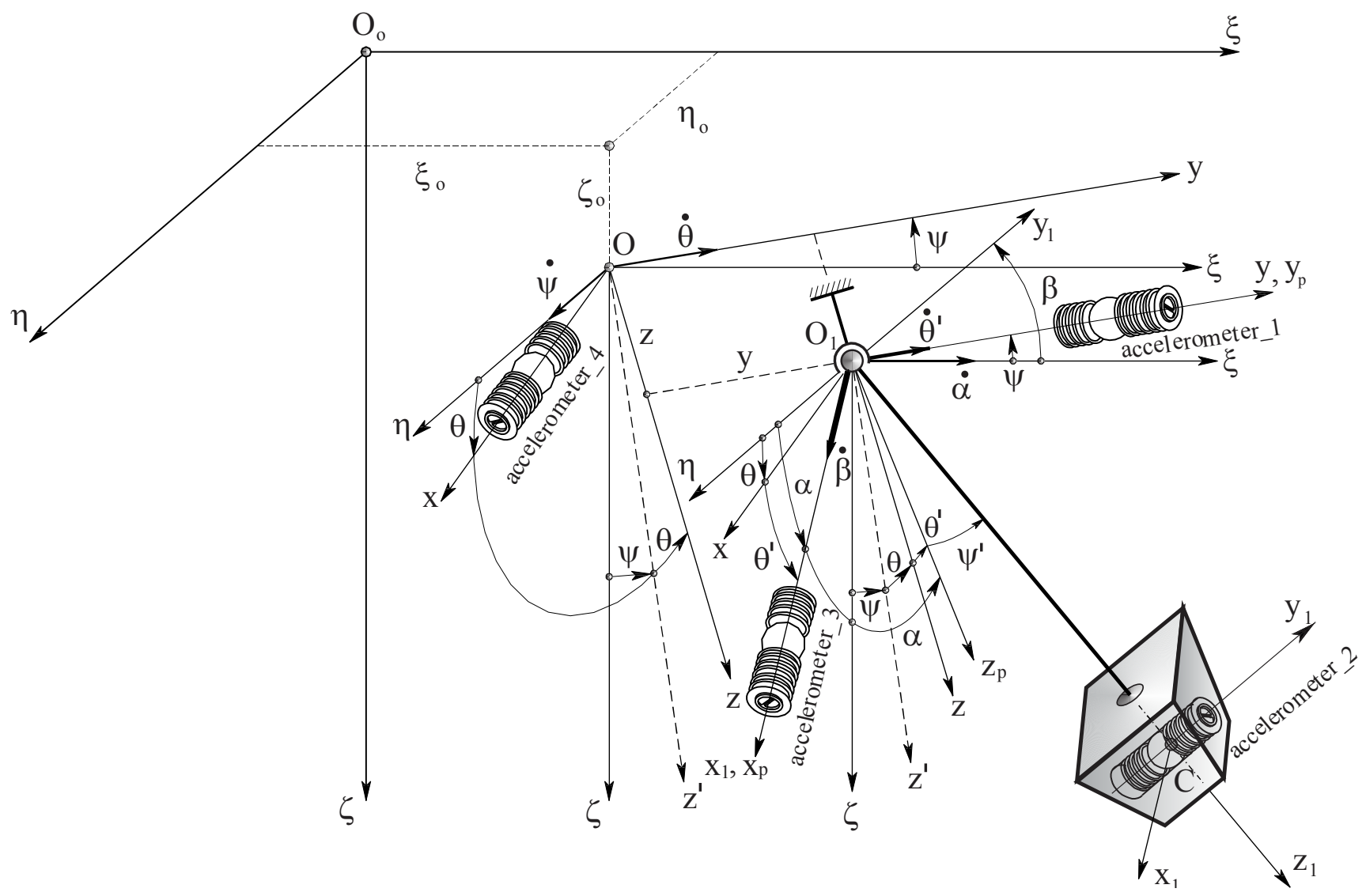

Fig.5. Diagram of the dynamic system moving object - measuring instrument.

The measurement accuracy in dynamic mode is ensured by an additional channel for determining the dynamic error. It consists of two pairs of identical MEMS accelerometers of SCA2100-D01 type used for measuring the linear acceleration. The accelerometers are mounted, respectively, on the body of the mechanical module, on the first cylindrical joint (two accelerometers), and on the physical pendulum (Fig.2). The first two accelerometers are mounted in such a way that their measurement axes are sensitive to the accelerations generated by the roll whereas the measurement axes of the other two accelerometers are sensitive to the accelerations generated by the pitch. This scheme of mounting of the MEMS sensors ensures the sensitivity of the first accelerometer of each sensor pair to all accelerations generated by the roll and the pitch. Every second accelerometer is sensitive not only to the accelerations of the first sensor but also to those generated by the pendulum motion in relation to its degree of freedom. This makes possible the development of a procedure involving the subtraction of the signals from the first and second accelerometer of each sensor pair, where the output signals are proportional to the accelerations generated by the pendulum motion in relation to its degree of freedom. By means of a data processing algorithm a double integration of the accelerations is performed, where signals defining the pendulum deviations from the vertical in relation to its two degrees of freedom are obtained.

Usually the measuring instruments operating in similar mode are subjected to other interference actions whose sources are external or internal additional secondary processes of unpredictable behavior. Hence, it is necessary to introduce an additional procedure for protecting the measuring instrument against the presence of these interference sources. Taking into account the characteristics of the quantities constituting the measurement environment where the measuring instruments under consideration operate, it can be concluded that the best form of eliminating the influence of the interference sources is the Kalman filter. The characteristics of this algorithm fit very well into the solution of a number of problems emerging in the process of optimization of the accuracy characteristics of the measuring instruments defining the parameters of the above listed moving objects $[8,10,13,14,17]$. Therefore, a module for signal processing by means of the Kalman algorithm is included in each measurement channel of the block diagram (Fig.2.).

\section{A MATHEMATICAL MODEL OF THE MEASURING SYSTEM}

The differential equations related to the motion of the instrument inertial components present the most complete description of the dynamic characteristics of the instruments. The equations are worked out for operating conditions close to the real ones. In order to make the mathematical operations easier, only one of the two measurement channels will be viewed - that related to the trim. In addition, the mathematical model of the accelerometer mounted on the cylindrical joint can be easily deduced from the equations of the second accelerometer 
positioned on the instrument pendulum, due to which it will not be taken into account when working out the differential equations.

The differential equations are worked out on the basis of a dynamic system shown in Fig.5. The motions of a ship are defined as angular and linear fluctuations of a rigid body around or along its center of gravity. The moving object (the ship), to which the coordinate system Oxyz is connected, changes its position randomly in relation to the supporting system $O_{o} \xi \eta \zeta$. The measuring instrument is mounted on the ship and its sensor (the physical pendulum) is connected to the coordinate system $C x_{1} y_{1} z_{1}$. The suspension point $O_{1}$ of the instrument sensor coincides with the diametral plane of the ship and its position with regard to the center of gravity of the moving object $O$ is defined by the $\mathrm{z}$ and $\mathrm{y}$ coordinates. The position of the moving object in relation to the supporting system $O_{o} \xi \eta \zeta$ is set by the three coordinates of its center of gravity $O-\xi_{o}, \eta_{o}, \zeta_{o}$ and the matrix $A=\left\|a_{i j}\right\|$ $(i, j=1,2,3)$ of the given angle cosines of the trim $\psi$ and the heel $\theta$, defining the angular displacement between the axes of the systems $O_{o} \xi \eta \zeta$ and $O x y z$. The mechanical system consists of two bodies - a physical pendulum which is free to rotate with regard to the coordinate axes $O_{1} x_{1}$ and $O_{l} y_{l}$, and an accelerometer mounted in the center of gravity $C$ of the physical pendulum. The accelerometer inertial body of a mass $m_{2}$ stays at an equilibrium position in relation to the $y_{1}$ coordinate by means of two horizontal springs of an elastic constant $c$.

Therefore, the mechanical system has three degrees of freedom and the generalized coordinates are, respectively, $\alpha, \beta$ and $y_{l}$. The $\alpha$ and $\beta$ coordinates define the angular displacement of the physical pendulum from the vertical in relation to, respectively, $O_{I} y_{1}$ and $O_{I} x_{1}$ axes whereas $y_{1}$ determines the relative motion of the inertial mass $m_{2}$. By means of the $\beta$ coordinate the inertial component of the dynamic error for the measurement channel under consideration is defined. The latter determines the trim values of a ship.

Upon working out the differential equations, the Lagrangian method is used. Their matrix form is of the following type:

$$
\begin{aligned}
& \mathbf{J} \cdot \mathbf{x}_{1}+\text { B.x } \mathbf{x}_{2}+\text { C.x } \mathbf{x}_{3}+\text { D. } \dot{\alpha} \cdot \dot{\beta}+ \\
& +\mathbf{M} \cdot \mathbf{x}_{\mathbf{4}}+\mathbf{G} \cdot \dot{y}_{1} \cdot \dot{\beta}+\mathbf{Q} \cdot \mathbf{y}_{\mathbf{0}}+\mathbf{H} \cdot \dot{y}_{1} \cdot \dot{\alpha}+ \\
& +\mathbf{K} \cdot \mathbf{y}+\mathbf{U} \cdot y_{1} \cdot \dot{\alpha} \cdot \dot{\beta}=\mathbf{S} \cdot \mathbf{z}_{\mathbf{0}}+\mathbf{E} \cdot \mathbf{z}_{\mathbf{1}}+ \\
& +\mathbf{F} . \mathbf{z}_{2}+\mathbf{N} \cdot \dot{\theta} \cdot \dot{\psi}+\mathbf{P} . \mathbf{z}_{3}+\mathbf{R} . \mathbf{z}_{4} \text {, }
\end{aligned}
$$

where $\mathbf{J}$ is a $3 \times 3$ square matrix with the inertial characteristics of the measuring system; $\mathbf{x}_{1}=\left\|\begin{array}{lll}\ddot{\alpha} & \ddot{\beta} & \ddot{y}_{1}\end{array}\right\|^{T} ; \mathbf{B}-$ a $3 \times 3$ matrix with the kinematic parameters of the system; $\mathbf{x}_{\mathbf{2}}=\left\|\begin{array}{lll}\dot{\alpha}^{2} & \dot{\beta}^{2} \quad \dot{y}_{1}{ }^{2}\end{array}\right\|^{T} ; \mathbf{C}-\mathbf{a}$ $3 \times 3$ matrix with the damping parameters of the system; $\mathbf{x}_{\mathbf{3}}=\left\|\begin{array}{lll}\dot{\alpha} & \dot{\beta} & \dot{y}_{1}\end{array}\right\|^{T} ; \mathbf{D}-$ a column vector $3 \times 1 ; \mathbf{M}-$ a $3 \times 3$ matrix with the mass characteristics of the system; $\mathbf{x}_{4}=\left\|\alpha \quad \beta \quad y_{1}\right\|^{T} ; \mathbf{G}-$ a column vector $3 \times 1 ; \mathbf{Q}-$ a $3 \times 3$ matrix with parameters characterizing the dynamics of the measuring system; $\quad \mathbf{y}_{\mathbf{0}}=\left\|y_{1} \cdot \ddot{\alpha} \quad y_{1} . \ddot{\beta} \quad y_{1} \cdot \ddot{y}_{1}\right\|^{T} ; \mathbf{H} \quad$ - a column vector $3 \times 1 ; \mathbf{K}-$ a $3 \times 3$ matrix with parameters characterizing the dynamics of the measuring system; $\mathbf{y}=\left\|y_{1} \cdot \dot{\alpha}^{2} \quad y_{1} \cdot \dot{\beta}^{2} \quad y_{1} \cdot \dot{y}_{1}^{2}\right\|^{T} ; \mathbf{U}$ - a column vector $3 \times 1 ; \mathbf{S}$ - a $3 \times 3$ matrix with parameters characterizing the dynamics of the ship; $\mathbf{z}_{\mathbf{0}}=\left\|\begin{array}{lll}\ddot{\eta}_{o} & \ddot{\xi}_{o} & \ddot{\zeta}_{o}\end{array}\right\|^{T} ; \mathbf{E}-$ a $3 \times 2$ matrix characterizing the inertial parameters of the "ship-measuring

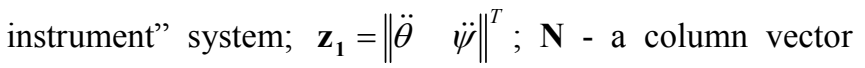
$3 \times 1$.

The differential equations (1) are worked out by taking into consideration the most complete and precise model of quantities, defining the measurement environment. Therefore, these differential equations describe in the most appropriate way the properties and characteristics of the measuring system. The availability of the mathematical model (1) allows us to forecast the measurement results when the system runs under different operating conditions, as well as to optimize its structure and parameters so as to satisfy the condition of a minimum error in the measurement result.

Reducing the equations (1) to a simplified form by means of linearization and inclusion of only small first-order quantities provides a clear picture of the concept used in the metrological procedure of the measuring system, i.e.

$$
\mid \begin{aligned}
& \left(J_{y_{l}}+m_{l} \cdot l^{2}\right) \cdot \ddot{\alpha}+k_{\alpha} \cdot \dot{\alpha}+m_{1} \cdot g \cdot l \cdot \alpha= \\
& =m_{l} \cdot l \cdot \ddot{\eta}_{o}-\left(J_{y_{l}}+m_{l} \cdot l \cdot z\right) \cdot \ddot{\theta} ; \\
& \left(J_{x_{l}}+m_{l} \cdot l^{2}\right) \cdot \ddot{\beta}+k_{\beta} \cdot \dot{\beta}+m_{l} \cdot g \cdot l \cdot \beta= \\
& =-m_{l} l \cdot \ddot{\xi}_{o}-\left(J_{x_{l}}+m_{l} \cdot z \cdot l\right) \cdot \ddot{\psi} ; \\
& m_{2} \cdot \ddot{y}_{l}+k_{y_{l}} \cdot \dot{y}_{l}+c \cdot y_{1}= \\
& =-\frac{1}{2} m_{2} \cdot\left(l \cdot \ddot{\beta}+\ddot{\xi}_{0}+z \cdot \ddot{\psi}\right)
\end{aligned}
$$

where $k_{\alpha}, k_{\beta}$ and $k_{y_{1}}$ are damping factors along the three generalized coordinates $\alpha, \beta$ and $y_{l} ; l$ - the length of the physical pendulum.

The third equation in (2) defines the link between the readings of the accelerometer mounted on the physical pendulum and the quantities entering at the input of the instrument. A differential equation representing the motion of the sensitive element of the second accelerometer can be easily worked out from this equation, taking into account the identical design characteristics of the two sensors and the fact that this accelerometer is not sensitive to the motion of the physical pendulum. Then the differential equation sought will be:

$$
m_{2} \cdot \ddot{y}_{p}+k_{y_{p}} \cdot \dot{y}_{p}+c \cdot y_{p}=-\frac{1}{2} m_{2} \cdot\left(\ddot{\xi}_{0}+z \cdot \ddot{\psi}\right)
$$


where $y_{p}$ is the coordinate of the motion of the sensitive element of the second accelerometer.

It can be seen in the third equation in (2) and (3) that the difference between the readings of the two accelerometers will be proportional to the function $\ddot{\beta}(t)$, by means of which we can easily determine the quantity $\beta(t)$ defining the dynamic error. Namely this mathematical model serves as a basis of the above proposed structure of the additional channel and the processing algorithm using the signals from two pairs of identical MEMS accelerometers.

\section{EXPERIMENTS}

To carry out the experiments, the required stand equipment has been developed. It is a hexapod of six degrees of freedom, which makes possible the reproduction of the fluctuations of the ship in a form close to the real operating conditions $[15,16,18]$. To ensure accuracy, the equipment is calibrated and metrological traceability of its unit to the length standard is provided. The equipment is shown in Fig.6.

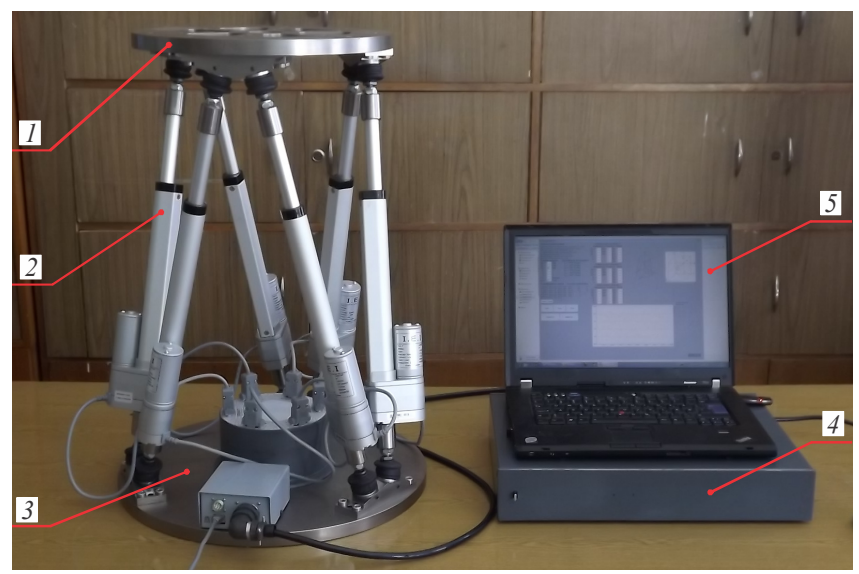

Fig.6. Stand equipment. 1 - operating platform; 2 - actuators; 3 immobile base; 4 - connection and control interface; 5 - computer.

Fig.7. illustrates the results from the investigation of the dynamic accuracy of the measuring system in its two operating modes. Within the first operating mode a Kalman filter is not used. Unlike within the second operating mode a module processing the measurement signals in compliance with the Kalman algorithm is used. The curves in Fig.7. show the dynamic errors for both operating modes in a graphic format. The errors, respectively, without $\varepsilon_{d e}(t)$ and with $\varepsilon_{d e}^{k f}(t)$ a module using a Kalman filter, are determined by the expressions

$$
\begin{aligned}
& \varepsilon_{d e}(t)=\psi_{m r}(t)-\psi(t) ; \\
& \varepsilon_{d e}^{k f}(t)=\psi_{m r}^{k f}(t)-\psi(t),
\end{aligned}
$$

where $\psi_{m r}(t)$ and $\psi_{m r}^{k f}(t)$ are the functions obtained as a result of the measurement of the platform motion, respectively, without and with a Kalman filter; $\psi(t)-$ the function defining the motion of the operating platform in relation to the trim coordinate.

Errors $\varepsilon_{d e}(t)$ and $\varepsilon_{d e}^{k f}(t)$ are time functions since according to (4) and (5) both the measurement result and the referent quantity are dynamically changing processes. Function $\psi(t)$ defining the referent motion of the operating platform along the trim coordinate is obtained as a result of the constitutive motions of the platform along its six degrees of freedom. The specific motion of the operating platform, upon measuring of which errors $\varepsilon_{d e}(t)$ and $\varepsilon_{d e}^{k f}(t)$ in Fig 7. are determined, is shown in Fig.8.

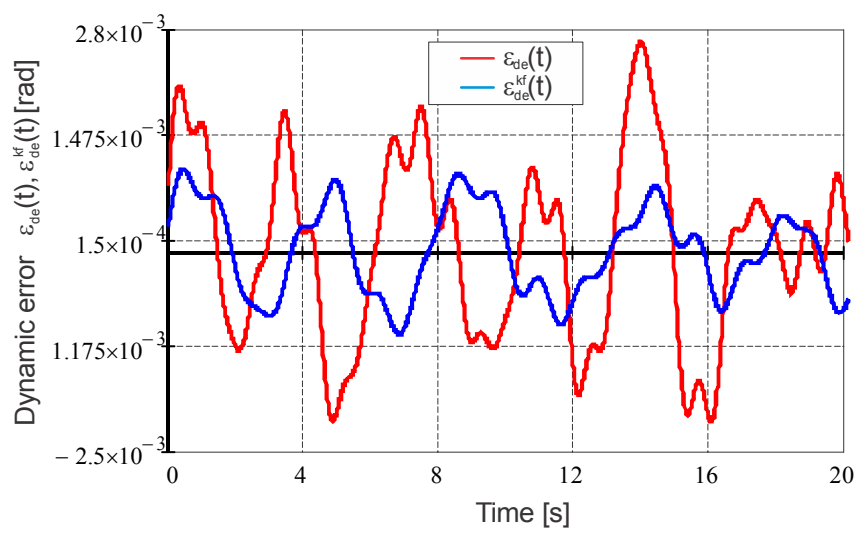

Fig.7. Results from the investigation of the dynamic accuracy.

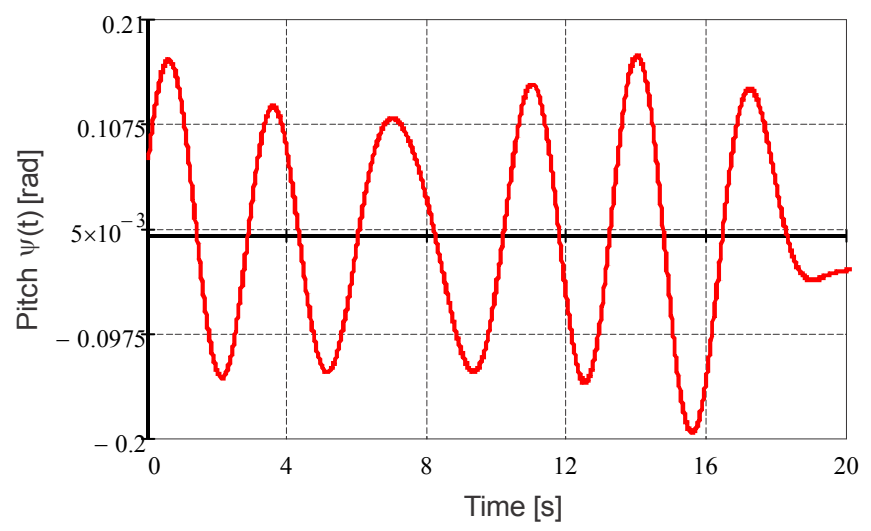

Fig.8. Motion of the operating platform along the angular coordinate $\psi$.

As it can be seen in Fig.7. (the red curve), the measuring system is stable enough in relation to its dynamic accuracy even without using the Kalman algorithm. In this case the maximum values of the dynamic error are within a range of $0.15^{\circ}-0.16^{\circ}$. The experiments show that even under the most severe conditions caused by the fluctuations of the ship the error does not exceed $0.2^{\circ}-0.3^{\circ}$.

The Kalman algorithm considerably improves the dynamic accuracy of the measuring system, which is illustrated in Fig.7. (the blue curve). It can be seen that the maximum values of the dynamic error $\varepsilon_{d e}^{k f}(t)$ vary within a range of $0.05^{\circ}-0.07^{\circ}$. 


\section{CONCLUSIONS}

The proposed measurement concept is designed for developing gyro-free measuring systems that determine the parameters of moving objects. This modeling approach overcomes the disadvantages of the existing measuring instruments since it is based, on one hand, on a very simplified mechanical module, and on the other hand, on the advanced achievements in the area of nanotechnologies, microprocessor and computer equipment.

The high dynamic accuracy of the proposed measuring system is ensured by an additional measurement channel operating in parallel with the main channel. The metrological procedures in the additional channel are based on an appropriate correction algorithm using signals from linear MEMS accelerometers.

The experimental results confirm the effectiveness of the proposed measurement concept in relation to the dynamic accuracy of systems measuring moving objects. As a result of the operation of the additional channel and the Kalman algorithm the accuracy characteristics of the measuring system under conditions of dynamic influences are improved to a great extent. This can be implemented without using expensive elements and stabilization systems.

\section{ACKNOWLEDGMENT}

The research reported in this paper was supported in part by project LO1201 funded by the Ministry of Education, Youth and Sports in the framework of the targeted support of the "National Programme for Sustainability I" and the OPR\&DI project Centre for Nanomaterials, Advanced Technologies and Innovation CZ.1.05/2.1.00/01.0005, as well as by Project M1309/2014 financed by the Scientific Research Fund at the Technical University of Gabrovo.

\section{REFERENCES}

[1] Dichev, D.A., Koev, H.C. (2012). Increase of dynamic accurasy in measurement systems of parameters of moving objects. In Metrology and Metrology Assurance : XXII National Scientific Symposium. Sazopol, Bulgaria, 57-64.

[2] Rivkin, S.S. (2002). Definition of Dynamic Errors of Gyro-Instruments on a Moving Base. Moscow: Azimut.

[3] Ivanov, Y.V. (2000). Autonomous sensors for heal, trim and vertical displacements of underwater and above-water objects. Sensors and Systems Magazine, 5 (1), 33-37.

[4] Pelpor, D.S. (1982). Orientation and Stabilization Gyroscopic Systems. Moscow: Mashinostroene.

[5] Belyanin, L.N., Yakimova, E.V. (2000). Vertical accelerometer plotter. In Electronic and Electromechanical Systems and Devices : XVI Scientific Symposium. Tomsk, 148-153.
[6] Danilov, A.T. (2001). A gyroscopic measuring system for parameters of moving objects. Problems of Special Machinebuilding Magazine, 4 (1), 178-181.

[7] Ivanov, Y.V. (2003). Inertial measurements in dynamic mode by means of high-frequency physical pendulums. Precision Engineering Magazine, 46 (9) 56-60.

[8] Albarbar, A., Badri, A., Sinha, J., Starr, A. (2009). Performance evaluation of MEMS accelerometers. Measurement, 42 (5), 790-795.

[9] Dichev, D., Koev, H., Louda, P. (2014). A measuring system with an additional channel for eliminating the dynamic error. Journal of Theoretical and Applied Mechanics, 44 (1), 3-20.

[10] Zhu, R., Sun, D., Zhou, Z., Wang, D. (2007). A linear fusion algorithm for attitude determination using low cost MEMS-based sensors. Measurement, 40 (3), 322328 .

[11] Dichev, D., Koev, H., Bakalova, T., Louda, P. (2014). A model of the dynamic error as a measurement result of instruments defining the parameters of moving objects. Measurement Science Review, 14 (4), 183189.

[12] Sveshnikov, A.A., Rivkin, S.S. (1974). Probability Methods in the Applied Gyroscope Theory. Moscow: Nauka.

[13] Venkatesh, K. Arun, Mathivanan, N. (2013). CAN network based longitudinal velocity measurement using accelerometer and GPS receiver for automobiles. Measurement Science Review, 13 (3), 115-121.

[14] Bohner, M., Wintz, N. (2013). The Kalman filter for linear systems on time scales. Journal of Mathematical Analysis and Applications, 406 (2), 419-436.

[15] Balchanowski, J. (2014). Some aspects of topology and kinematics of a 3D of translational parallel mechanism. International Journal of Applied Mechanics and Engineering, 19 (1), 5-15.

[16] Kazakoff, A. (2012). Advances in engineering software for lift transportation system. Journal of Theoretical and Applied Mechanics, 42 (1), 3-22.

[17] Dichev, D., Koev, H., Bakalova, T., Louda, P. (2013). An algorithm for improving the dynamic accuracy in systems for measuring the parameters of moving objects. International Journal of Engineering, Business and Enterprise Applications, 5 (2), 1-9.

[18] Pendar, H., Mahnama, M., Zohoor, H. (2011). Singularity analysis of parallel manipulators using constraint plane method. Mechanism and Machine Theory, 46 (1), 33-43.

Received April 23, 2014. Accepted October 02, 2014. 